\title{
Improved approaches of automated lung segmentation on digital tomosynthesis images
}

\author{
Bence Tilk \\ Budapest University of Technology and Economics 1111 Budapest, Müegyetem rkp. 3., Hungary
}

\begin{abstract}
For lung screening the most common method is chest radiography, which produces summation images without giving any depth information about the lung. Computed Tomography (CT) creates excellent slice images, which give volume data that makes CT a more sensitive nodule detection system. However, CT has disadvantages, it is too expensive and its x-ray emission is too high to be used as an everyday screening method. Digital tomosynthesis (DTS), as a relatively new chest imaging modality, can be positioned between chest radiography and CT. While it produces slice images of the chest similarly to $\mathrm{CT}$, its slice thickness is larger, it creates a bit more blurred slices, it has much lower radiation than CT. This blurring makes it hard to segment the lung areas automatically, which is essential for an efficient Computer-aided Diagnosis system. The paper proposes a combined method, which starts from a previously published approach, extends it using snake methods and adjacent images' segmentation information to improve lung segmentation. Experiments show that the combination of methods reduces the incorrectly segmented lung region.
\end{abstract}

\section{Section: RESEARCH PAPER}

Keywords: medical imaging; digital tomosynthesis; lung segmentation; computer-aided diagnosis

Citation: Bence Tilk, Improved approaches of automated lung segmentation on digital tomosynthesis images, Acta IMEKO, vol. 6, no. 4, article 7, December 2017, identifier: IMEKO-ACTA-06 (2017)-04-07

Section Editor: Alexandru Salceanu, Technical University of lasi, Romania

Received May 5, 2017; In final form October 9, 2017; Published December 2017

Copyright: @ 2017 IMEKO. This is an open-access article distributed under the terms of the Creative Commons Attribution 3.0 License, which permits unrestricted use, distribution, and reproduction in any medium, provided the original author and source are credited

Corresponding author: Bence Tilk, e-mail: bence.tilk@gmail.com

\section{INTRODUCTION}

Lung cancer is one of the leading causes of death. Early detection of the lung nodules can increase the survival chance of the patient. The most common screening method used to inspect the lungs is chest radiography, which produces single summation images. These $\mathrm{x}$-ray images do not give in-depth information about the lung, so abnormalities like lung nodules cannot easily be separated from other anatomical shadows like vessels, bones, etc. Although computed tomography (CT) is a much more sensitive imaging modality, its cost and the radiation dose a patient obtains during a CT scan are obstacles for largescale screening use. These drawbacks are true even for its low dose version (LDCT). Chest digital tomosynthesis (DTS), as a relatively new imaging modality, can be positioned between chest radiography and CT [1]. While it produces (coronal) slice images of the chest similarly to CT, its slice thickness is larger, it creates a bit more blurred slices, and the radiation dose produced during a DTS scan is much lower than that of a CT scan. A further consequence of using DTS is that, instead of a single $\mathrm{x}$-ray image, a DTS scan produces about $100-400$ coronal slice images. So, reading the images of a complete scan needs more time and thoughtfulness from the radiologists/pulmonologists.

Computer-aided Diagnosis (CAD) can help physicians, can improve radiologists' efficiency as it detects nodule-like areas, and helping radiologists to decide if this area can be regarded as a potential abnormality or not.

A modern CAD system has 3 main parts. To create Regions of Interest (ROIs), robust methods are needed to segment the lungs from other parts of the image, and to determine areas of the images where any abnormality is looked for. The second part is to find suspicious regions inside the previously segmented area. The third part is to classify the candidate nodules with machine learning tools and to provide a list of potential lesions which can be overlooked by doctors.

This paper focuses on the first step of the procedure. It proposes a complex lung segmentation method of DTS slice images. DTS is considered as a $2.5 \mathrm{D}$ imaging technique. It creates projections similar to standard x-ray radiography, however, instead of getting a single projection of a patient, 40-60 projections are taken from different angles. From these 
projections reconstruction algorithms are used to compute the coronal slice images of the chest. Because the projections are taken from a limited angle range, the coronal slices are not ideally thin, and there is some blurring between the neighbouring slices too. Although the spatial resolution of the images is rather good (the coronal slices are at least $1000 \times 1000$ pixel images) their in-depth resolution is poor making difficult to obtain $3 \mathrm{D}$ information about the lung. Central slices have relatively high quality, but further from the central slice, image quality is getting poorer, what can mislead the segmentation methods on non-central images. This paper proposes a complex segmentation procedure, which starts from a method published in [2], but as the result of this previous method does not seem satisfactory, further steps are introduced. The complex algorithm applies three different approaches: an intensity-based, a gradient-based and an energy-based segmentation in order to make the algorithm more robust and accurate. The efficiency of the method is increased by combining information from the adjacent images.

This paper is organized as follows: Section 2 is a short summary of the previous results; it describes the most promising solutions and shows their deficiency. In Section 3. the improvements of the previous method are described. An evaluation is presented in Section 4, where simulated DTS images are used, which are generated from CT images, to get at least acceptable standard reference lung contours.

\section{RELATED RESULTS IN THE LITERATURE}

DTS is a relatively new medical imaging modality. Its main application is related to breast screening [3], while only a few products can be found where DTS is used for chest screening [4]. Previous results that are dealing with computer-aided detection or diagnostic (CAD) systems based on DTS are even fewer. According to the knowledge of the author, there are only very few papers dealing with the problem of lung segmentation in chest DTS slice images. Seung-Hoon et al. [5] proposes segmentation using only intensity information with a region growing method on central images. This paper recommends using a previously selected fixed pixel as an inner point, which is the initial point of the growing method. This approach is not considered as a robust method because it is sensitive to the position of the patient. It also suggests rolling-ball, where a ballshaped element is rolled along the edge of the segmented area to get smooth lung borders in the end, as a useful postprocessing step.

Automated lung segmentation in chest DTS [2] uses the gradient information of the image and searches the lung masks along the edges by dynamic programming. The gradient image is transformed into polar coordinates where an inner point determined previously is used as the origin of the polar coordinate system. This approach works quite well in the central slice images, as in these images the lung area has rather definite and sharp borders. This paper also proposes a method on how to handle non-central images. The basic idea is that the neighbouring slices are rather similar, so the gradient images in these slices must be similar too.

The results using these approaches are quite remarkable, however, the robustness of these methods should be improved. As the variability of DTS images obtained from different patients is rather large, the performance of these methods varies greatly.

A typical gradient image of a DTS slice is shown in Figure 1

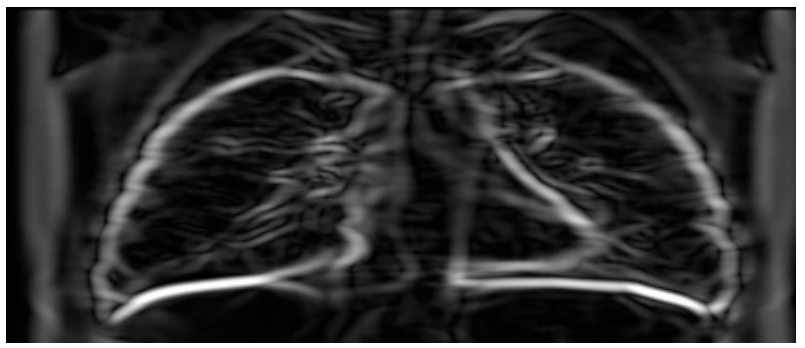

Figure 1. Gradient image of one DTS slice in the Cartesian coordinate system; it is squeezed along the vertical dimension to set each lung's width and height to have similar sizes. This step is done in order to eliminate as many jumps on the path as possible.

and its version in the polar coordinate system is shown in Figure 2. As it can be seen, there are brighter paths and many pale paths in the image, and these paths are going through the gradient image near horizontally, as the lung contour, where there may be larger gradients, surrounds the inner point.

In this unfolded image, dynamic programming is used to find a route connecting the brightest points. The drawback of this algorithm is that it assumes that one column of this image belongs to one angle, that is, only convex shapes can be identified using dynamic programming. A cost function is assigned to each pixel, which has two components. One of them is the intensity of the pixel; it is used as a reward. The second component is the distance difference from the previous pixel; this is used as a penalty. More details about the cost function are available in [2]. The result of the method for a slice is shown in Figure 3.

Concave segments may show up around the heart, so when using this approach the area under the heart can't be segmented correctly and additional methods are required to find this

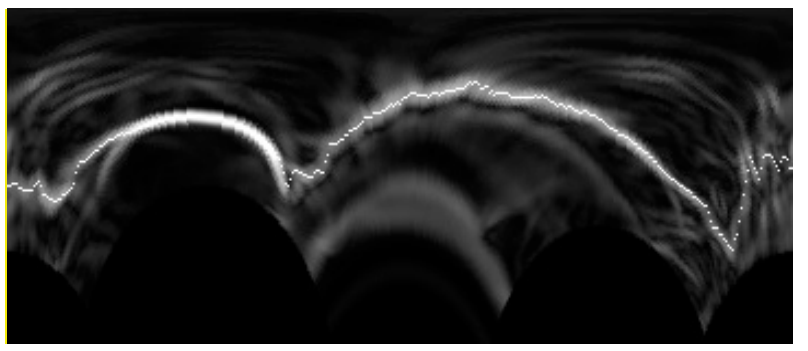

Figure 2. Gradient image of one DTS slice in the polar coordinate system; white pixels mark the calculated path. Only the right lung is shown in this image.

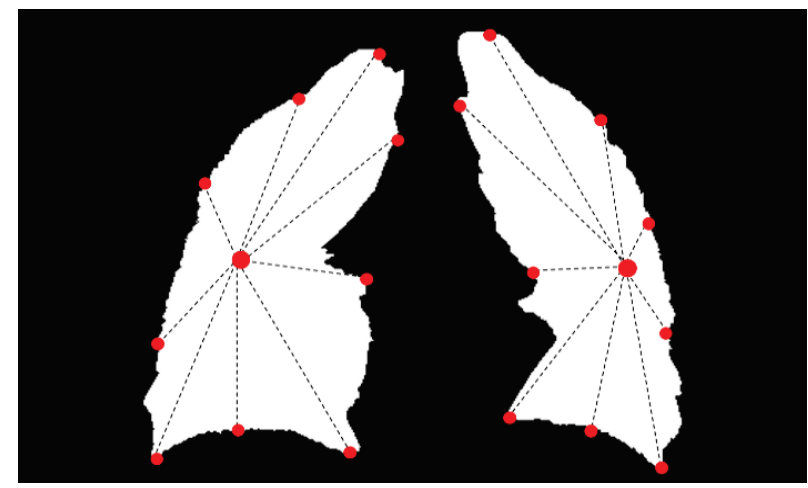

Figure 3. After reconstructing the found path, the center of the lung and some points are highlighted with red dots. 
region. There is another disadvantage of dynamic programming. Two paths can be very different while they may have similar costs. The consequence is that there may be very similar slice images, but the segmented masks can be significantly different, what makes this method less robust.

This study [2] also suggests a method to reduce wrong segmentation on non-central slice images, by fading unlikely position pixels on the polar coordinate images, as the assigned positions on the adjacent slices must be close to each other. On the current gradient image, the pixel values are multiplied by a Gaussian function, where pixel distances from the adjacent selected points in that column are assigned as Gaussian function's variable, see (1).

$I^{\prime}(x, y)=I(x, y) G(O(x)-y)$

where $I(x, y)$ is the intensity value of one pixel of the polar coordinate image, at the $x$-th column and $y$-th row. $G$ is a Gaussian weighting function and $O(x)$ is the neighbouring row index in the gradient path, which image is towards the center slice. So $O(x)-y$ is a distance between the two selected pixels in the $x$-th column. Images are processed from the center slice towards the non-central slices.

This model eliminates problems in many cases, but sometimes a wrong central image could mislead the whole process. It is more desirable to have more images around the central one instead of exactly one because a poorly chosen slice as a center image can reduce the accuracy. This is based on the assumption that, choosing such images where at least half of them are considered as blurred ones, is less likely.

An important and hard problem of lung border detection is to validate the results. The study [2] uses reference lung contours, which are created by radiologists. However the real lung contours in DTS slice images are very difficult to determine even for skilled radiologists. On the central slice images it is quite easy to determine the lung contour but on non-central images the expected lung area based only on DTS can hardly be determined. This is why more information is needed to get at least approximately correct lung borders on non-central images.

\section{MODEL-FREE METHOD}

\subsection{Pixel intensity based segmentation}

Using only pixel intensity values to determine the lung area is impossible as - in contrary to the CT images - on the DTS images the intensity values are not standardized. This means that on DTS images a universal intensity limit, correct for differentiating the lung area from non-lung areas on every image cannot be identified. Instead, picture-dependent limits should be defined. Pixels below this limit are considered as part of the lung. The correct value of the intensity limit can be determined by using the histogram of the image, which is shown in Figure 4 for a typical slice. First, the histogram is smoothed using a low-pass filter to reduce noise and the effect

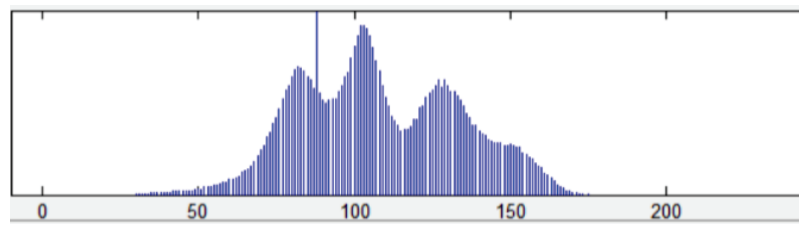

Figure 4. Original histogram of a chest tomosynthesis; some intensity values are missing. of missing intensity values. A simple averaging filter with a 5pixel kernel is used. The histogram has two valleys after filtering. A proper limit value in the smoothed histogram will be the local minimum of the first valley.

After that, morphological methods are used to get continuous lungs and to remove noise. Experiments show that eroding with a smaller structure-element and dilating with a bigger structure-element gives the best results.

Next, some inner point of each lung, called center point, must be determined. The image is split vertically at the center into two parts (the two (half) lungs), so one lung is processed by the method, then the formulas below are used; $P(x, y)$ is the value of the binary mask at pixel $(x, y)$ :

$$
\begin{aligned}
& \text { Center }_{x}=\sqrt{\sum_{(x, y)} x^{2} P(x, y)} \\
& \text { Center }_{y}=\sqrt{\sum_{(x, y)} y^{2} P(x, y)}
\end{aligned}
$$

Results show that this point isn't optimal because it is near to the arteries and the heart, which can be misleading for the further steps of the method. The centers are translated to the outer side of the lung by $8 \%$ of the image width and translated upwards by $10 \%$ of the image height. (An example of the selected center points is shown in Figure 5).

\subsection{Gradient-based segmentation}

The next step is using a path finder algorithm. Due to the previously mentioned problem of ambiguity of getting a path, additional smoothing is needed. Get every point of the lung contour on each slice and make a function from it; the values of the function will be the distances from the central point. Generate these functions $-F_{x}(y)$ belongs to the $x$-th point of the boundary and $y$ is the index of the slice, as can be seen in Figure 6 -, then convolve the function as described in (4) with a smoothing filter ( $\mathrm{H}$ kernel, which can be any vector; the chosen one is described in (5)), so it decreases the big steps on the masks between the adjacent images. After the convolution,

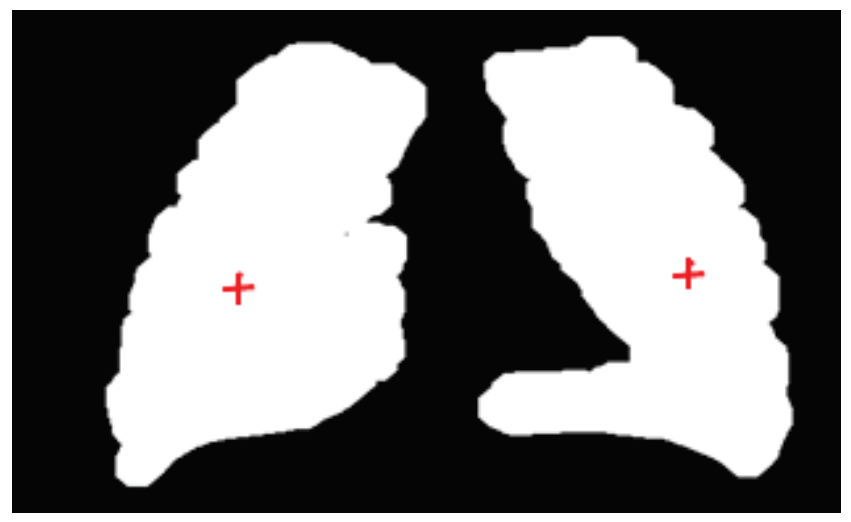

Figure 5. Segmented area based on pixel intensity, after morphological steps; red dots are the calculated centers.

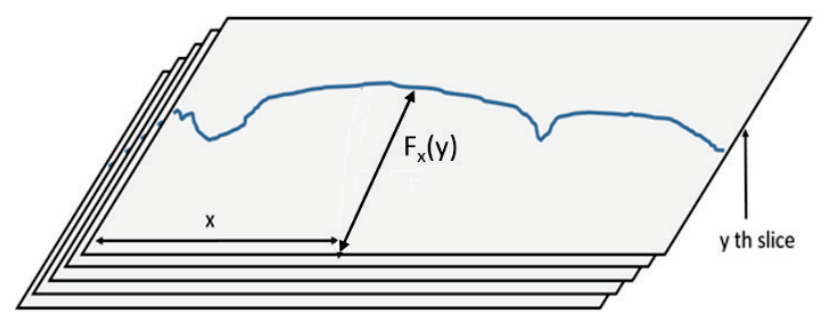

Figure 6. Construct $F_{x}(y)$ function based on images in polar coordinates. 
convert the values back to the Cartesian coordinate system to get the suggested path.

$F_{x}^{\prime}(y)=F_{x}(\mathrm{y}) * H$

$H=\frac{1}{5}[1 ; 1 ; 1 ; 1 ; 1]$

In the end, the presented method works almost perfectly on the outer side of the lung, but it gives rather bad results near the mediastinum.

\subsection{Energy based segmentation}

Fortunately, there is a method that works better on the inner side of the organ: we can use an active contour model (sometimes referred to as Snake) to find the region under the heart on the left lung and it also works more or less on the mediastinum. The active contour model [6] is based on energy minimization; it creates smooth contours and, at the same time, it takes the edges into consideration as can be seen in Figure 7.

The energy formula is the sum of the smoothness of the contour, the continuity of the contour (first two components known as internal energy) and image energy. Furthermore, with correct parametrization on images that differ slightly, it creates almost identical masks. It is an iterative method and needs an initial mask. An intensity-based segmentation is considered as a satisfying initial mask. It contains the region under the heart, but it isn't accurate enough.

To combine the two methods, use the active contour model to define the inner side of the lung and use the gradient-based method to determine the others. The regions are determined by the range of angles and the chosen inner point is used as a center point.

\subsection{Information from adjacent slices}

The information of adjacent images can be used to fit the segmentation to obtain more reliable masks. The basic method [2] is modified to get a better model. The image in polar coordinates is modified, fading the unlikely pixels. Unlike the method in [2], two Gaussian functions are used. The first one gives a weight to the adjacent images, which depends on how far it is from the calculated image; only the images to the central direction are relevant, weights in other directions are zero. The second Gaussian function fits vertically to the image like in the referred article. The center of the function is the path of the adjacent image in the selected pixel of the current column. Here the main modification of the function is: using an asymmetric Gaussian function (shown in Figure 8), with a lower width

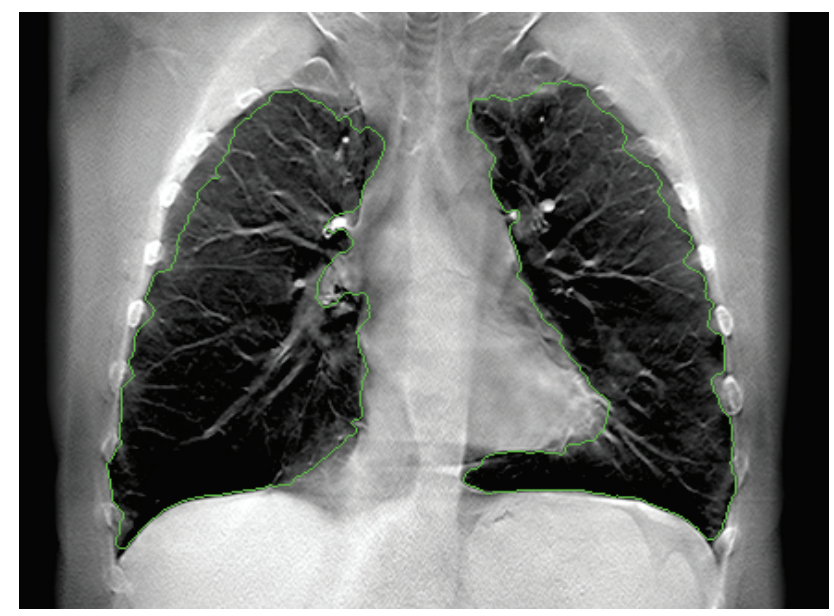

Figure 7. Active Contour result after 30 iterations.

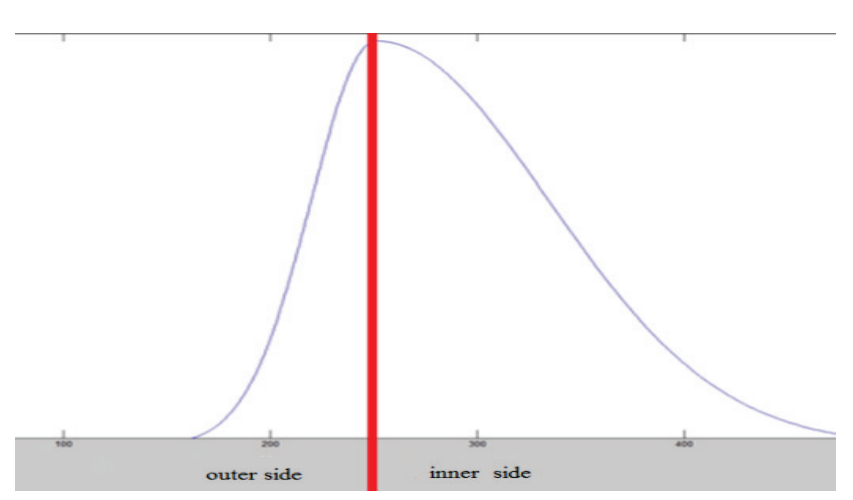

Figure 8. Asymmetric Gauss function, with different standard deviation on each side; both sides are normalized to give the same results in the origin.

parameter on the farther side from the origin and a higher $\sigma$ on the nearer side.

This approach is used because the lung area is bigger on central slices and it shrinks when going further from the central slice. $I(x, y)$ is the current pixel, $N$ is a constant for normalization, $G$ is a standard Gaussian function, $G_{2}$ is the asymmetric Gaussian function and $O(x, \imath)$ is the $x$-th point of the found path on the $i$-th image:

$I(x, y)=N \sum_{i} I(x, y) G(i) G_{2}(O(x, i)-y)$

In the end, the masks may contain acute angles. It is desirable to minimise them using morphological closing to accomplish this. Usage of different structure sizes on different regions of the lung gives the best result The biggest structure is used at the inner side of the lungs and a smaller one used on the top of the lungs; the lower area is skipped completely. This approach produces similar results as those of the rolling ball, but with less computational complexity [5].

\section{MODEL-BASED IMPROVEMENTS}

Correct segmentation of the lung can be interpreted as a measurement with error. The measurement error can be reduced as the expected distribution of the measurement is known. In order to make a correct segmentation, prior knowledge is needed about shape, size, the location of the lung and characteristics of tomosynthesis. To build a model the reference shapes of the lungs on each slice are needed. Section 5 of this paper shows the method of generating these references from an image set [7]. Because the generated references are on simulated images, features of the images cannot be used because it may add a bias to the model. Considering these facts, the final method is an extension of the snake algorithm [6]. It adds size and shape energy to the model as can be seen the in (7), where $x$ iterates through the boundary.

$$
\begin{aligned}
& E_{\text {snake }}=\int_{0}^{1}\left(E_{\text {internal }}(x)+E_{\text {image }}(x)+E_{\text {shape }}(x)+\right. \\
& \left.E_{\text {size }}(x)\right) \mathrm{d} x
\end{aligned}
$$

The snake algorithm minimizes the energy; the internal energy describes the smoothness of the shape and the image energy describes how well the shape fits to the image edges. To optimize the energy of the boundary, the gradient or its approximation must be provided as well.

Modelling the size of the lung on each slice can be done by modelling the relative size of the lung on each slice. The relative size of an average left lung on each slice can be seen in Figure 9. The model stores the relative size of the segmentation, 


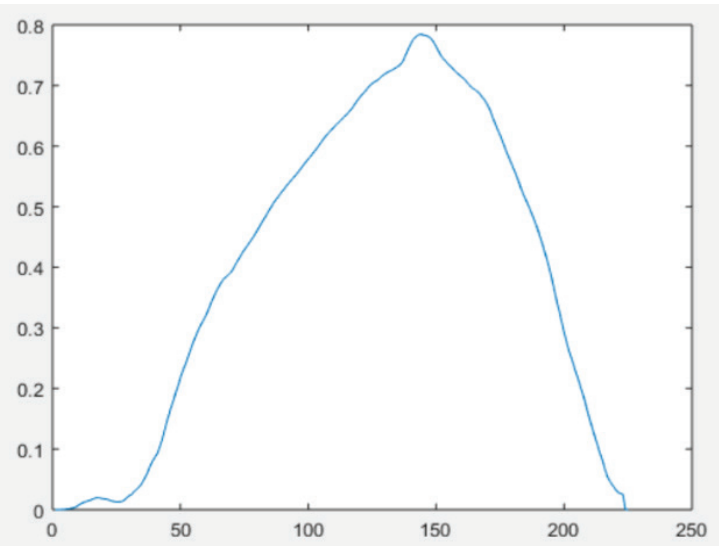

Figure 9. The relative size of the lung on each slice: the $x$-axis shows the ordinal number of the slice, and the relative (compared to the biggest seen lung area) size of the lung area is shown on the $y$-axis. This graph is calculated from an average of 8 lungs. 250 slices are used, many of them don't contain lung volume. The peak on the graph is caused by the area of the apex.

compared to the chosen interval of the slices. The central interval is used as the reference size and other slices are compared to it because its size is the easiest to determine with model-free methods.

The size energy is calculated on each slice as the absolute difference of the relative reference lung size and the current relative size (compared to the central images) of the segmentation. Of course the size energy on the central interval is zero. Equation (8) declares $\boldsymbol{\alpha}$, which is a normalization coefficient. It shows the size of the patient's lung compared to the reference lung. $C_{\text {start }}$ and $C_{\text {end }}$ are the endpoints of the interval of central slices, $M_{\text {start }}$ and $M_{\text {end }}$ are the same for the modelled reference lung:

$\alpha=\frac{M_{\text {end }}-M_{\text {start }}}{C_{\text {end }}-C_{\text {start }}} * \frac{\sum_{i=C_{\text {start }}}^{C_{\text {end }}} \operatorname{Size}(\operatorname{Image}(i))}{\sum_{i=M_{\text {start }}}^{M_{\text {end }}} \operatorname{ModelSize}(i)}$

Considering the model lung and the current image set have the same number of slices, (9) shows the size energy on the current slice:

$E_{\text {size }}(x)=|\alpha \operatorname{ModelSize}(i)-\operatorname{Size}(\operatorname{Image}(i))|$

The gradient of the size energy directs towards the normal vector of segmentation; its magnitude equals the calculated size energy. The shape energy is calculated as DTW (Dynamic Time Warping) distance of two time series [8]. Time series are generated for each lung on each slice; an example for this time series is shown in Figure 10.

DTW is a dynamic programming technique, calculated as below. $D T W(i, j)$ means the cost of time series one $\left(t_{1}\right)$ till the $i$-th point and time series two $\left(t_{2}\right)$ till the $j$-th point. The cost of two values is calculated as absolute distance. The value of the DTW function is 0 if the value of $i$ or $j$ equals zero.

$\operatorname{DTW}(i, j)=\operatorname{cost}\left(t_{1}(i), t_{2}(j)\right)+\min \{\operatorname{DTW}(i-1, j)$,

$\operatorname{DTW}(i, j-1), \operatorname{DTW}(i-1, j-1)\}$

One of these time $\left(t_{1}\right)$ series is the reference series on the given slice, and the other $\left(t_{2}\right)$ is the calculated one from the current boundary and normalized to have the same average for both time series. The boundary is transformed to time series similarly as the image in polar coordinates is calculated. The same center point is used but this time the distance for every

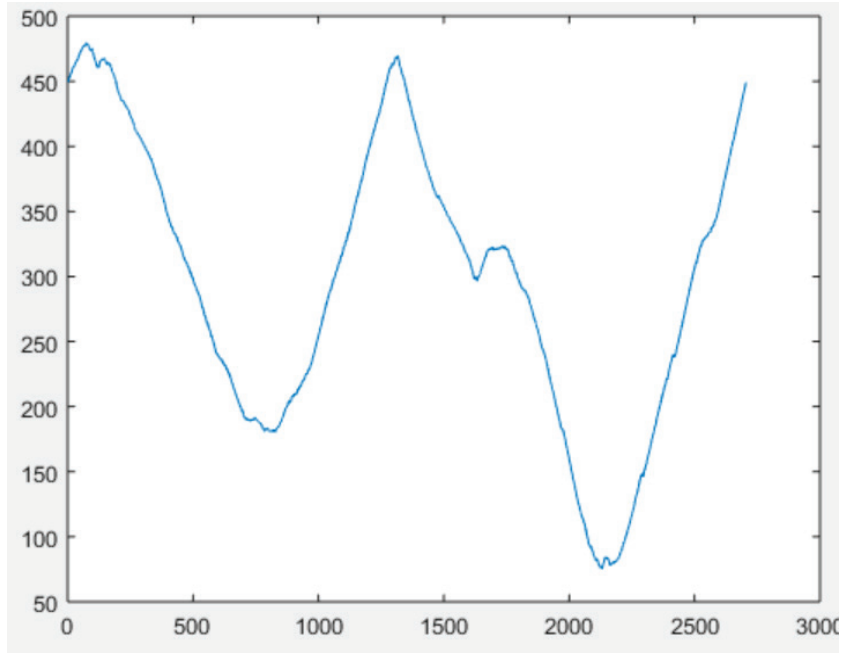

Figure 10. This time series is generated from the left lung on the 130th slice of 250 slices. More than 2500 boundary points are used and the distance is measured in pixels: the $x$-axis shows the ordinal number of the boundary point and the pixel distance of the boundary point from the center point is shown on the $y$-axis.

point of the boundary is calculated by iterating the points from a starting point to a given direction. This method creates a time series shown in Figure 10. The shape energy can be calculated according to the above information and an approximate gradient is needed for the iterative method. The equations below show how to calculate the gradient. Equation (11) describes the magnitude of the gradient for the $i$-th point. Equation (12) shows the gradient calculation: the first part shows the gradient direction, which directs inwards and its magnitude is 1 ; the third part decides whether it should direct inwards or outwards.

$|\nabla \mathrm{P}(\mathrm{i})| \approx \operatorname{cost}\left(t_{1}(i), t_{2}(j)\right)$

$\nabla \mathrm{P}(\mathrm{i})=\frac{\left(\begin{array}{l}O_{x} \\ O_{y}\end{array}\right)-\left(\begin{array}{l}P_{x}(i) \\ P_{y}(i)\end{array}\right)}{\left\|\left(\begin{array}{l}O_{x} \\ O_{y}\end{array}\right)-\left(\begin{array}{l}P_{x}(i) \\ P_{y}(i)\end{array}\right)\right\|} *|\nabla \mathrm{P}(\mathrm{i})| *$

$*\left\{\begin{array}{cc}\text { if } t_{1}(i)>t_{2}(j): & 1 \\ \text { else: } & -1\end{array}\right\}$

\section{RESULTS}

Validation of the results for non-central slices is challenging, where blurring could mislead radiologists, so without any further information lung contours created by them cannot be considered as reliable segmentation. This problem can be partially solved with simulated tomosynthesis images, which are generated from axial CT slices. The simulated image characteristics are close to those of the original DTS images. On axial CT images, as can be seen in Figure 11, lung masks are generated based on pixel intensity and morphological closing. As a next step, these images were projected to coronal slices with similar geometry as the geometry of the DTS. Masks are projected as well; they are used as an at least acceptable standard reference, which is useful to calculate metrics too.

\subsection{Center slices}

Two metrics are calculated: overlapping and mean average distance (MAD). MAD is calculated as an average of each distance of discovered points to the nearest reference point. 


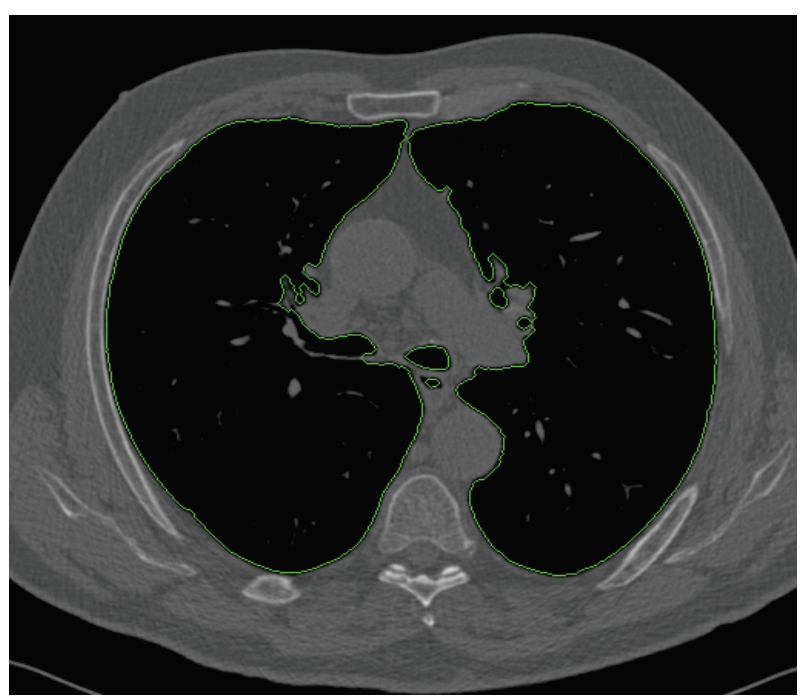

Figure 11. Axial CT slice segmented by the intensity of the pixel; the green line is separating lungs from other parts.

These standard deviations are calculated as well. Overlapping is calculated by:

$\frac{\sum_{i, j} R(i, j) \cap M(i, j)}{\sum_{i, j} R(i, j) \cup M(i, j)}$

and the Mean Average Distance (MAD) is calculated by:

$\frac{1}{N} \sum_{i=1}^{N} \sqrt{\left(R_{x}(i)-M_{x}(i)\right)^{2}+\left(R_{y}(i)-M_{y}(i)\right)^{2}}$

In $(14) \mathrm{R}(i, j)$ denotes the pixel of the reference mask at the $i$-th row and $j$-th column and $M(i, j)$ is the segmented mask with the same parameters.

The evaluation is done by five central images of the image sets. The results are weighted by the number of central images. An image is considered as a central image if the cross-sections of the ribs are sharply visible. The number of central images on the used image set with 223 slices varies from 41 to 57 . The aggregated results are shown in Table 1.

Each image set of a patient has been investigated; results depend on images quality. Table 2 shows the results for the

Table 1. Averaged results using 5 central images of the image set. Image resolution is $512 \times 512$.

\begin{tabular}{lccl}
\hline Result & Overlap & MAD (pixel) & $\begin{array}{l}\text { Standard } \\
\text { deviation }\end{array}$ \\
\hline Both lungs & 0.7976 & 5.452 & 6.630 \\
Left lung & 0.8089 & 4.507 & 4.770 \\
Right lung & 0.7882 & 6.109 & 7.019 \\
\hline
\end{tabular}

Table 2. Results of one image set out of 57 central images from 223 images, 130 of them containing lung area according to CT images. Resolution of the images is $512 \times 512$ pixels.

\begin{tabular}{lccc}
\hline Result & Overlap & MAD (pixel) & $\begin{array}{l}\text { Standard } \\
\text { deviation }\end{array}$ \\
\hline Both lungs & 0.8508 & 5.175 & 5.943 \\
Left lung & 0.8462 & 5.125 & 6.117 \\
Right lung & 0.8557 & 5.169 & 5.510 \\
\hline
\end{tabular}

patient whose image set shows the least blurry.

Images are compared inside one image set. Figure 12 shows a segmentation of a central image, Figure 13 shows a segmentation of a non-central image from the same image set. The next section provides more information about the accuracy trend inside one image set.

\subsection{Result on noncentral images}

Table 3 shows the results for non-central slices; central borders have been identified manually.

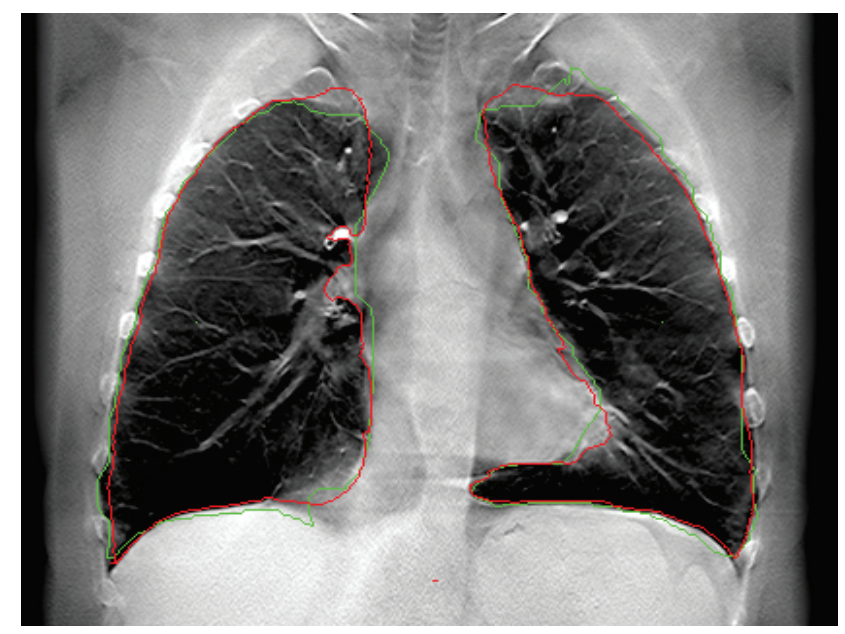

Figure 12. On a central image successful segmentation, the green line is the method's boundary and the red one is the reference lung, which comes from a segmented CT-scan.

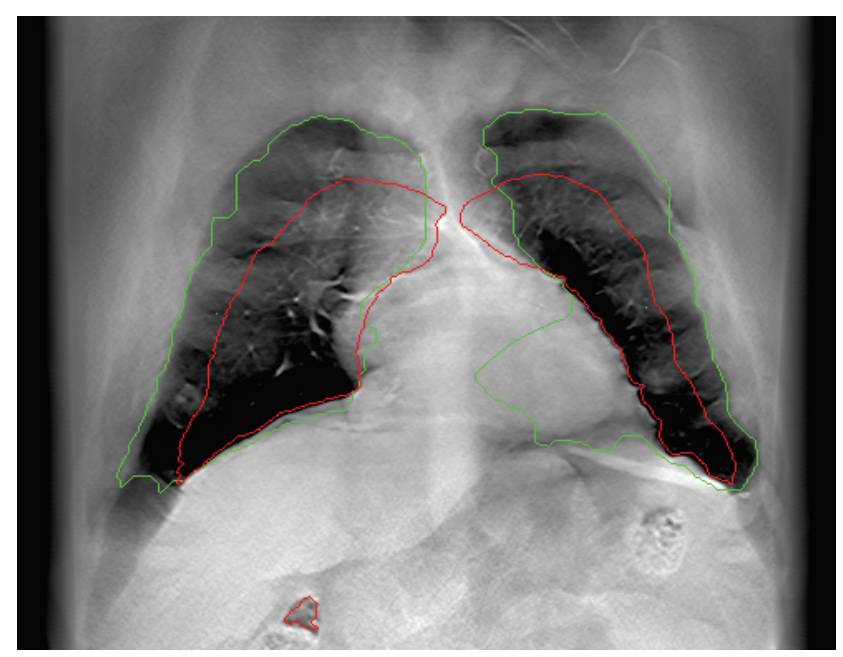

Figure 13. On a simulated non-central slice, the red line is the reference lung based on $\mathrm{CT}$; it is hard to be separated by radiologists without any help.

Table 3 Segmentation with a model on non-central images, which contain lung area.

\begin{tabular}{lll}
\hline Result & Overlap & MAD (pixel) \\
\hline Average & 0.4922 & 20.529 \\
Standard deviation & 0.2027 & 17.258 \\
Max & 0.8314 & 109.485 \\
Min & 0 & 3.744 \\
\hline
\end{tabular}


Figure 14 shows the difference between the model-based and the model-free approach. It suggests that modelling lung features improve the results on non-central images and create more robust segmentation on these slices. That is why model results tested only on non-central slices. Results are calculated from 8 image sets each of them containing 225 slices Only those slices are used that contain lung area. Figure 15 shows sample lung segmentations on various slices.

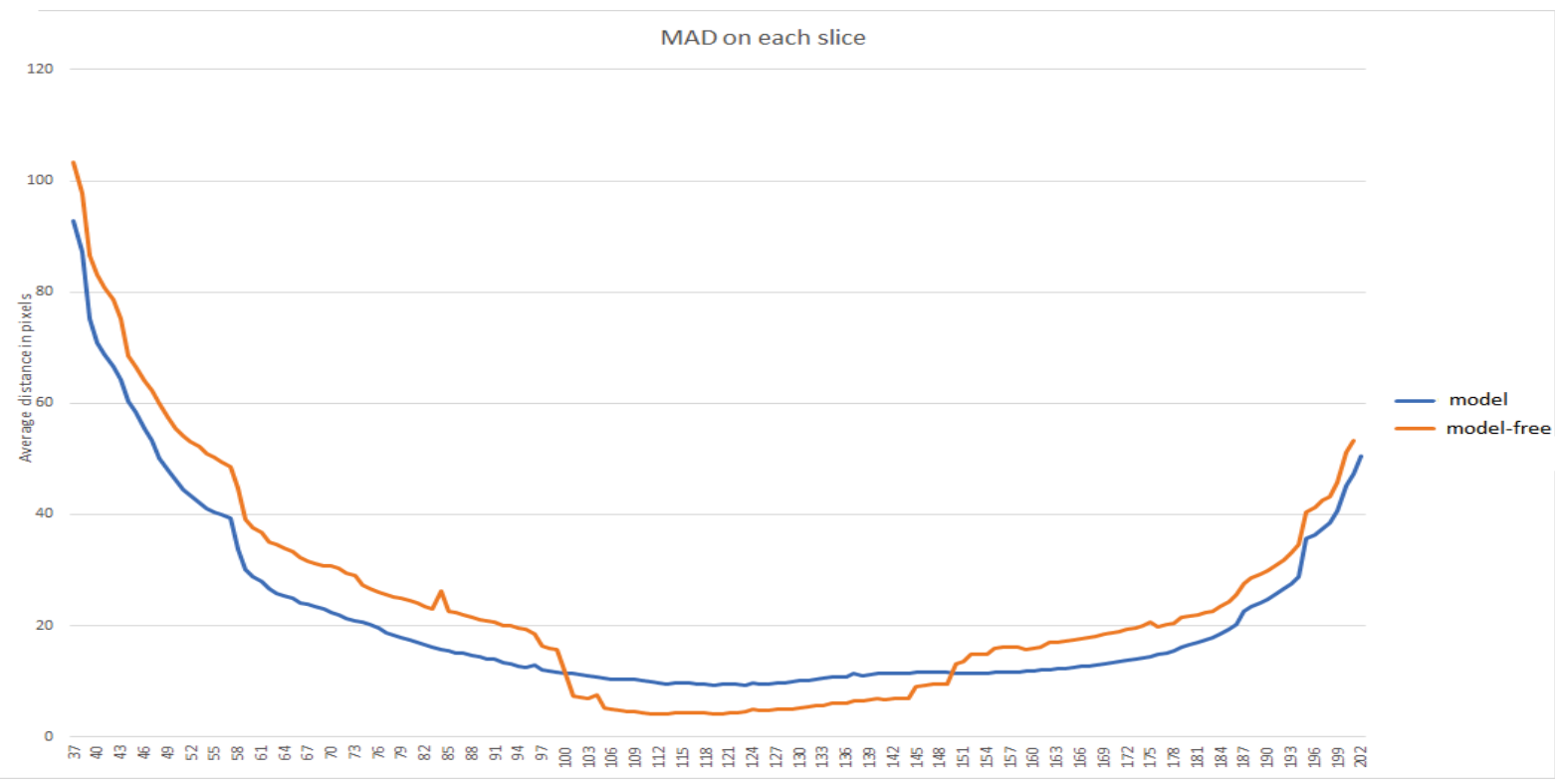

Figure 14. Model-based and model-free approaches comparison based on MAD. The method with a model is more robust, but on central slices the image itself is more reliable than the model suggested segmentation.

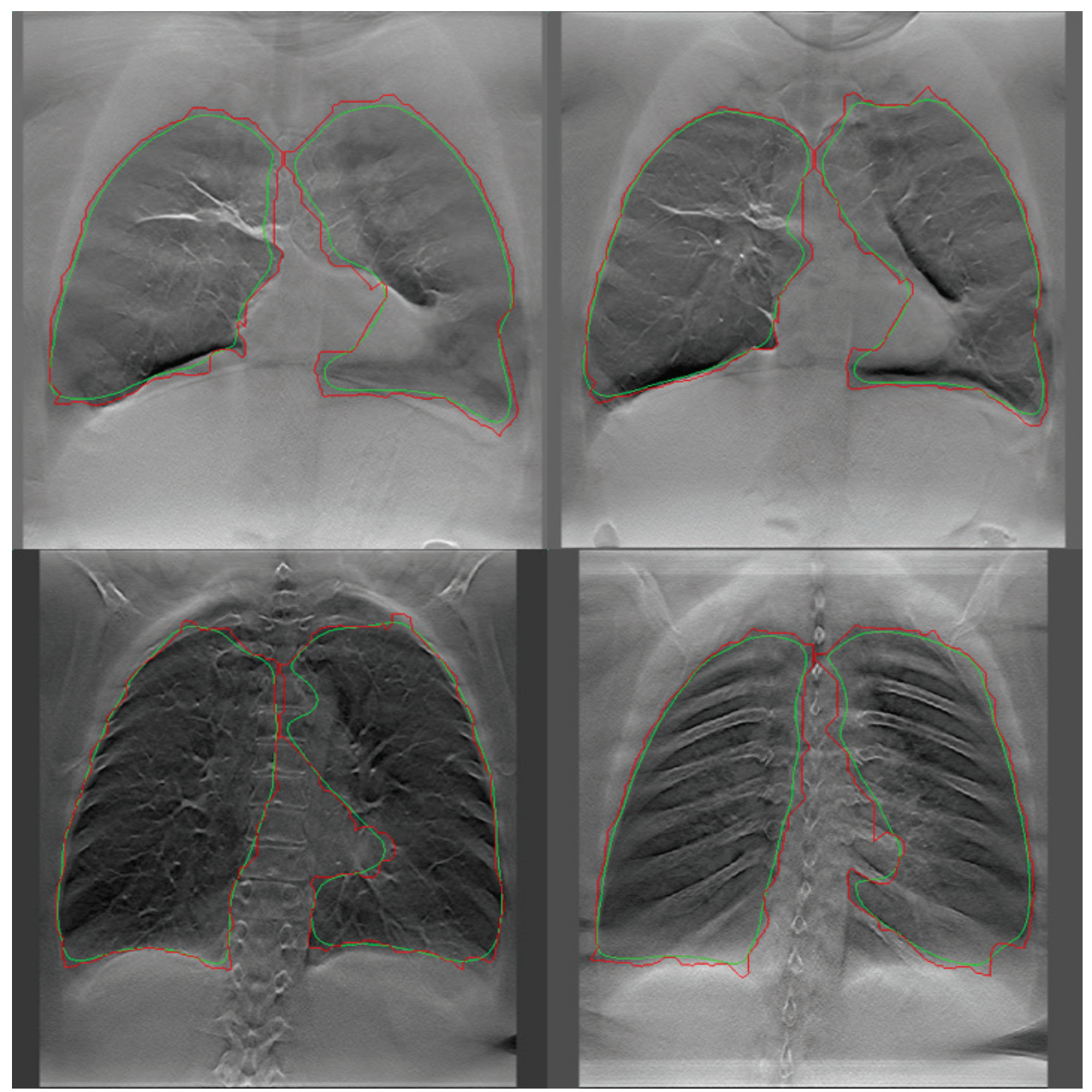

Figure 15. Segmentation on digital tomosynthesis images; red is the model-free version, the green line is the segmentation with a model. Image's order is anterior-posterior. The method using the model seems more natural for humans. 


\section{CONCLUSION}

A combined method can improve the results and may result in more robust solutions. Three different model-free approaches are used to take advantage of each method on different areas of the lungs: intensity-based segmentation for center point identification, gradient-based segmentation for segment outer side of the lung and snake for segment outer side of the lung. Using more complex models is to gain information from adjacent images to improve the incorrectly segmented region on the non-central images.

The inspection mostly focused on the central images, because without them, non-central slices cannot be accurately segmented, and most of the information is concentrated on central images; blurring through adjacent images here can be useful. It is proved that further improvement can be reached on non-central images by creating a model of the lung using statistical methods. This paper showed two statistical methods to model the lung's size and shape. These methods can increase accuracy on non-central slices and make the numbers slightly worse on central images. Combining these two approaches can produce the highest accuracy, but models have many difficulties. One of the biggest problems is the large number of images that are required to get a correct model.

\section{ACKNOWLEDGEMENT}

The author thanks the help of Gábor Horváth, who introduced the problem, and Dániel Hadházi for generating simulated tomosynthesis images from LIDC CT images [7].

\section{REFERENCES}

[1] J.T. Dobbins, "Tomosynthesis imaging: at a translational crossroads", Medical physics, 36, (2009), pp.1956-1967.

[2] Wang, Jiahui, J.T. Dobbins, Q. Li, "Automated lung segmentation in digital chest tomosynthesis", Medical physics, 39, (2012), pp.732-741.

[3] Park, M. Jeong, E. A. Franken, M. Gargand, L.L. Fajardo, and L.T. Niklason, 'Breast tomosynthesis: present considerations and future applications", RadioGraphics, 27, (2007), pp.231-240.

[4] J.T. Dobbins, H.P. McAdams, J.W. Song, C.M. Li, D.J. Godfrey, D.M. DeLong, S.H. Paik, and S. Martinez-Jimenes, "Digital tomosynthesis of the chest for lung nodule detection: Interim sensitivity results from an ongoing NIH-sponsored trial", Medical physics, 35, (2008), pp.2554-2557.

[5] C. Seung-Hoon, L. Jeongwon, W. Chulho, P.S. Bum, "Lung Segmentation Using Prediction-Based Segmentation Improvement for Chest Tomosynthesis", International Journal of Bio-Science and Bio-Technology, 6, (2014), pp.81-90.

[6] M. Kass, A. Witkin and D. Terzopoulos, "Snakes: Active Contour Models", International Journal of Computer Vision, 1, (1988), pp. 321-331.

[7] A. G. Samuel, G. McLennan and L. Bidaut, M.F. McNitt-Gray, C.R. Meyer, A.P. Reeves, B. Zhao, D.R. Aberle, C.I. Henschke, E.A. Hoffman, "The lung image database consortium (LIDC) and image database resource initiative (IDRI): A completed reference database of lung nodules on CT scans", Medical Physics, 38, (2011), pp.915-931.

[8] H. Sakoe, S. Chiba: "Dynamic programming algorithm optimization for spoken word recognition", IEEE transactions on acoustics, speech, and signal processing, 26, (1978), pp.43-49. 